Open Access : ISSN : 1848-7718

Review

\title{
An overview on the role of telomere, telomerase in degenerative diseases and cancer
}

\author{
Churong $\mathrm{Li}^{1}$, Yin Gang ${ }^{1}$ \\ ${ }^{1}$ Department of Radiotherapy, Si Chuan Cancer Hospital, Chengdu, Sichuan Province, China \\ *Corresponding Author: E-mail: cxqyguestc@163.com; Tel.: +86 028-85420305; Fax: +86 028-85420305
}

Received: August 07, 2015; Revised: August 20, 2015; Published: September 05, 2015

\begin{abstract}
Telomerase maintains the length of telomeric DNA at the end of chromosomes; stabilizes the functions of chromosomes, protects the structure of chromosome DNA and regulates normal cell growth. The length and stability of telomeres determine the cellular lifespan. Moreover, they correlate with cellular aging and carcinogenesis. Researches on the role of telomerase in degenerative diseases and cancers show that it regulates cellular senescence. Although telomerase is not an oncogene, it does make tumor cells immortal by maintaining the stability of telomeric DNA. Telomerase-based therapies have become a hotspot in new anticancer and degenerative diseases treatments. This review aims to provide a comprehensive understanding of the role of telomerase and telomere in cellular senescence and cancer.
\end{abstract}

\section{Keywords}

Telomerase; telomere; degeneration diseases; cancer

\section{Introduction}

Telomeres are specialized structures located at the terminal ends of chromosomes and play a critical role in stabilization of chromosomes, maintaining the integrity of the genome [1,2], and regulation of normal cell growth. Telomerase is a reverse transcriptase enzyme which synthesizes the G-rich tandem repeats that comprise telomeres using a template on the RNA that is complementary to the telomeric repeat. By adding hexameric (TTAGGG) repeats, the continued erosion of telomeres is compensated. Over the past few decades, studies have shown that telomeres and telomerase are closely related to cellular malfunction, degenerative diseases and tumor progression. Therefore, researches into the role of telomerase and telomere in degenerative diseases and cancer shed light on a better understanding of these diseases. Telomerase-based therapies, including targeted agents will open up a whole new horizon for degenerative diseases and cancer treatments.

\section{Telomeres, telomerase and their relationship}

Telomere, from the Greek word "telos," (end), and "meros," (part) was first named by American geneticist Hermann J. Muller, who observed through mutation experiments carried out during the 1930s [3]. Telomeres which have specialized structures that repeat at the ends of chromosomes are thought to be important for the integrity and stability of chromosomes. This observation implied that cells contained an 
enzyme that could catalyze the lengthening of chromosomal ends. In 1984, Elizabeth Blackburn discovered the enzyme telomerase in the ciliate Tetrahymena, which has the ability to lengthen chromosomal ends by its reverse transcriptase activity [4].

Telomeres DNA sequences are highly conservative and genus-species specific. Human telomeres contain an array of tandem DNA repeats (5'-TTAGGG-3'), which do not encode any gene product. The length of the telomere DNA repeats varies from about $5 \sim 15 \mathrm{kp}$. These special DNA repeats combine their associated proteins to form the cap of chromosomes, which can prevent chromosome end-to-end fusion, degradation and rearrangement from happening, unless the free ends of the DNA molecule are processed as double stranded breaks [5]. To avoid chromosome damage, the end of telomeres forms two internal loops (D-loop and T-loop) with the participation of the shelterin complex [6]. Usually the shelterin complex is made up of six proteins which play different roles, including telomeric repeat-binding factors 1 and 2 (TRF1 and TRF2), TRF1-interacting nuclear factor 2 (TIN2), repressor activator protein 1 (Rap1), TIN2-interacting protein 1 (TINT1) and protection of telomeres 1 protein (POT1). TRF1 and TRF2 bind to double stranded TTAGGG repeats, and the POT1 binds to the 3' G-rich overhang. The three remaining factors contained TIN2, Rap1 and TINT1, which interact with the telomere-bound proteins to form a complex. This allows cells to distinguish telomeres from sites of DNA damage [7-9]. Telomeres maintain the integrity of chromosomes. As the cell divides, about 100-200 bp of telomeric DNA sequence is lost. This phenomenon is so-called the "end replication problem". When the DNA repeats strand becomes too short to form loops, the chromosome loses its endpoint protection [9]. This is why the telomeric repeats shorten progressively with each cell division [10]. If the telomeres are shortened enough, telomerase is activated to compensate for the end-replication problem [11], so that the length of telomeres is maintained.

Human telomerase which is a ribonucleoprotein polymerase (RNP) composed of an essential RNA and associated proteins has important function. When chromosome loses its endpoint protection, telomerase is activated to catalyze the addition of TTAGGG nucleotide repeats to the $3^{\prime}$ terminus of the chromosome's DNA [12], so that it guarantees the length and function of telomeres. In generally, the telomerase holoenzyme consists of three components: telomerase reverse transcriptase (TERT), the telomerase RNA component (TERC) and telomerase related proteins (TEP). The TERT and TERC are most important. TERC as a template can add a TTAGGG nucleotide repeat to the $3^{\prime}$ end of the telomere's leading strand; TERT is taken to be the rate-limiting component of telomerase activity [13]. TERC varies greatly among species, but TERT is relatively conserved throughout evolution [14]. In humans, TERC contains 451 nucleotides, which includes an 11-nucleotide template and several conserved regions $[13,15]$. It can provide the template for the synthesis of telomere DNA repeats, and also plays essential roles in catalysis and accumulation of telomerase RNP. The TERT gene which located on chromosome 5, consists of 16 exons and 15 introns spanning $35 \mathrm{~kb}$, including 330 base pairs upstream of the translation start site, as well as 37 base pairs of exon 2 of the TERT gene. The TERT promoter which contains many sites for several transcription factors is GC-rich and lacks TATA and CAAT. It is an important target for therapy of cancer and degenerative diseases. Telomerase has other related proteins (TEP), such as GAR1, NHP2 and NOP10. They are assembled in the telomerase complex and play distinctive roles in telomerase function [16].

\section{The role of telomere and telomerase in degenerative diseases}

Telomere length shortening has been evidenced as not only a marker of biological aging but also a mechanism with important functional consequences. It has been considered as a risk factor for multiple aging-associated diseases and conditions. The study of telomere and telomerase biology shows a higher prevalence of development in many neurodegenerative diseases, such as Alzheimer's disease (AD), 
Parkinson's disease (PD), CVD, hypertension, atherosclerosis, heart failure, diabetes and so on. This review focuses on providing a general understanding of the role of telomere and telomerase in Alzheimer's disease (AD) and Parkinson's disease (PD).

Alzheimer's disease and Parkinson's disease are the two main aging-associated progressive neurodegenerative disorders. Studies show that they are related to telomere and telomerase. The relationship between telomere shortening and $A D$ has been studied using in vitro and in vivo models in $A D$ patients. Oxidative stress is regarded as the most important influencing factor of telomere shortening. There are some studies connecting the telomere length to Alzheimer's disease. Panossian L. A. et al. [17] first found that patients with $A D$ had shorter telomere lengths in peripheral blood mononuclear cells than that of the age-matched control subjects. Then, Jenkins E. C et al. [18] found that older females with Down syndrome and AD-type dementia had shorter T-cell telomeres than that of the age-matched counterpart. Moreover, a case control study of 257 individuals revealed a shortened peripheral blood leukocyte (PBL) telomere length in AD patients compared with control subjects [19]. And among individuals with AD, short telomeres were associated with higher mortality rates. Another study confirmed the association between short $\mathrm{PBL}$ telomere length and $\mathrm{AD}$, and was the first to examine hippocampus telomere length in histopathologically validated $A D$ patients [20]. Surprisingly, telomere lengths in the hippocampus were found to be $49 \%$ longer in $A D$ patients than in control subjects $(P<0.01)$. Recently, J. Nicholas Lukens compared the telomere lengths in peripheral blood and cerebellum in Alzheimer's disease and found that reduced PBL telomere length in AD might not reflect reduced telomere length in bulk brain tissue, but may be a marker of changes in a subset of brain tissues or other tissues that affect the pathogenesis of AD [21]. Telomerase activity in AD patients was significantly elevated compared with healthy controls and vascular dementia patients, possibly as a response to the telomere erosion accompanying the disease. In addition, the telomerase activity of lymphocytes was significantly correlated with the degree of dementia in $A D$ patients, suggesting an accelerated telomere dysfunction in lymphocytes and impaired immune function of AD patients.

The relationship between telomere and PD is not well clear. Several studies have shown that telomere and telomerase are related to PD, but other studies indicate that there are no evidence for short telomere length in PD. Wang et al. [22] proved that men with shorter telomeres had a lower risk for PD, which was in marked contrast with the observations in other aging-related diseases. In a study of 28 Japanese male PD patients, leucocyte telomere length in PD patients was not significant different from those in controls. But mean telomere length in PD patients was found to be $5 \mathrm{~kb}$ shorter suggesting telomere shortening was accelerated in PD patients [23]. Additionally, Maede et al. [24] investigated the age-associated alterations of subtelomeric methylation in Japanese PD patients. Short telomeres with hypomethylated subtelomeres increased with aging in the healthy controls, but did not change in the PD patients. Johanna Eerola et al analyzed 131 PD patients and 115 controls. It was found that extremely short telomeres were not more frequent in PD patients than in controls, so they consider there was no evidence for shorter leukocyte telomere length in PD $[25,26]$.

The role of telomere and telomerase in neurodegenerative diseases is not fully understood through above researches, despite the progress made during the last decade. Further research is essential to clarify whether the $A D$ and $P D$ are triggered by telomere shortening.

\section{The role of telomere and telomerase in cancer}

Telomere studies in human cancer have been extensively reported for diagnostics and prognostics. Shortened telomere length demonstrates a significant association with poor cancer outcome [27]. 
Telomere maintenance is regarded as an important mechanism in evading senescence by cancer cells, and the role of dysfunctional telomere biology in cancer is acknowledged. Many cancer cases were initiated by reactivating or up-regulating telomerase activity. In addition, in many types of cancer cell, there is a considerable shortening of telomeres despite telomerase expression [28-32]. Based on these researches, several anti-cancer drugs and cancer vaccines that target the telomerase are used now in phase III clinical trials.

The relations between telomere, telomerase and cancer have been a hot research topic. Cancers occur when a cell acquires multiple genetic mutations which cause the cell to escape from normal controls on replication and migration. Once the cells and their offspring multiply uncontrollably, they can invade and damage nearby tissue. Some may also break away and travel to parts of the body where they do not belong, resulting in new metastases at distant sites. The lack of telomerase in tumor cells may slow the growth with dividing cells, and lose their telomeres before they do much damage. On the other hand, if cancer cells were able to generate telomerase, they could retain telomeres and might growth indefinitely. Researchers suspect that the loss of proliferative capacity observed in human cells that lacking telomerase may help us to avoid cancer. Here, we direct our attention to digestive cancers, telomere and telomerase.

Telomerase activity is especially considered as the most important factor in carcinogenesis, progression and prognosis. Fundamental researches showed that telomerase was activated in various cancer tissues and cell lines [33-35]. Telomerase activity was shown to be positive in $85 \% \sim 100 \%$ of cancer tissues and negative in almost all normal tissues, except for reproductive and haemopoietic cells. In digestive cancers, many studies regard telomerase activity and telomere length are associated with the occurrences of cancer, and as prognostic markers in malignancies. Studies indicate telomerase activity is detected in $85 \%$ of the hepatocellular carcinoma specimens [36], $85 \%$ \%8 \% of gastric carcinomatous tissues [37], and 90 $\%$ of colorectal tumors [38]. In gastrointestinal cancer, human telomerase are expressed in various levels between all tumor specimens and normal mucosa. For instance, the expression of telomerase is $81 \%$ higher in the tumor than normal mucosa. Meanwhile $35 \%$ of normal mucosa showed telomerase activity and all of them contained intestinal metaplasia [39,40]. The study by Engelhardt $M$ indicated that increased telomerase activity occurs in colon cancer cells that have undergone extensive telomere shortening relative to surrounding normal tissues, in which telomerase-induced stabilization of telomeres may be critical for the continued proliferation of the malignant clone [38]. The link between telomerase activity and stage suggests that telomerase is up-regulated as a function of increased tumor cell invasion, tumor progression, and metastatic potential in colon cancer. On the research of prognosis, telomere length could be a potential unique biomarker predictive of clinical benefit (i.e. progression free survival) of $\mathrm{mCRC}$ patients treated with anti-EGFR therapy [41]. The high level of telomerase activity may be an independent prognosis-predicting factor in the patients with colorectal cancer [42].

\section{Prospect}

To sum up, the related research shows that the occurrence and changes of degenerative diseases is closely related to telomere shortening. Especially, the study for patients with Alzheimer and Parkinson shows that the dysfunction of telomerase activity and telomere shortening which may lead to the occurrence of these disease and relate to the severity of the disease. Through the study of telomerase activity, it may be possible to find new ways of treatment for these two diseases. In addition, there are studies to explore the relationships and related mechanisms between tumor and telomere, telomerase. Telomerase activation and telomere shortening are closely related to the occurrence and development of malignant tumor. Currently, the study of telomerase targeted drugs for the treatment of cancer has 
entered the phase III clinical trials. Hopefully findings from these studies will emerge as a new approach for cancer therapy.

\section{References}

[1] R.K. Moyzis, J.M. Buckingham, L.S. Cram, M. Dani, L.L. Deaven, M.D. Jones, J. Meyne, R.L. Ratliff, J.R. Wu, Proc. Natl. Acad. Sci. USA 85 (1988) 6622-6626.

[2] E.H. Blackburn, Nature 350 (1991) 569-573.

[3] H.J. Muller, The collecting net 13 (1938) 181-198.

[4] C.W. Greider, E.H. Blackburn, Cell 43(2Pt1) (1985) 405-413.

[5] M.G. Ferreira, K.M. Miller, J.P. Cooper, Mol. Cell. 13 (2004) 7-18.

[6] D. Li, Q. Yuan, W. Wang, J. Int. Med. Res. 40 (2012) 1242-1250.

[7] D. Liu, M.S. O'Connor, J. Qin, Z. Songyang, J. Biol. Chem. 279 (2004) 51338-51342.

[8] T. de Lange, Genes Dev. 19 (2005) 2100-2110.

[9] U. Herbig, W.A. Jobling, B.P. Chen, D.J. Chen, J.M. Sedivy. Mol. Cell 14 (2004) 501-513.

[10] J.D. Watson, Nat. New Biol. 239 (1972) 197-201.

[11] W.E. Wright, M.A. Piatyszek, W.E. Rainey, W. Byrd, J.W. Shay, Dev. Genet. 18 (1996) 173-179.

[12] C.W. Greider, E.H. Blackburn, Cell 43 (1985) 405-413.

[13] C. Autexier, N.F. Lue, Annu. Rev. Biochem. 75 (2006) 493 - 517.

[14] Q. Zhang, N.K. Kim, J. Feigon, Proc. Natl. Acad. Sci. USA 108 (2011) 20325-20332.

[15] J. Feng, W.D. Funk, S.S. Wang, S.L. Weinrich, A.A. Avilion, C.P. Chiu, R.R. Adams, E. Chang, R.C. Allsopp, J. Yu, et al., Science 269 (1995) 1236-1241.

[16] K. Collins, J.R. Mitchell, Oncogene 21 (2002) 564-579.

[17] L.A. Panossian, V.R. Porter, H.F. Valenzuela, X. Zhu, E. Reback, D. Masterman, J.L. Cummings, R.B. Effros, Neurobiol. Aging. 24 (2003) 77-84.

[18] E.C. Jenkins, M.T. Velinov, L. Ye, H. Gu, S. Li, E.C. Jenkins Jr, S.S. Brooks, D. Pang, D.A. Devenny, W.B. Zigman, N. Schupf, W.P. Silverman, Neurobiol. Aging. 27 (2006) 941-945.

[19] L.S. Honig, N. Schupf, J.H. Lee, M.X. Tang, R. Mayeux, Ann. Neurol. 60 (2006) 181-187.

[20] P. Thomas, N.J. O'Callaghan, M. Fenech, Mech. Ageing Dev. 129 (2008)183-190.

[21] J. Nicholas Lukens, V. van Deerlin, C.M. Clark, S.X. Xie, F.B. Johnson, Alzheimers Dement. 5(6) (2009) 463-469.

[22] H. Wang, H. Chen, X. Gao, M. McGrath, D. Deer, I. de Vivo, M.A. Schwarzschild, A. Ascherio, Mov. Disord. 23 (2008) 302-305.

[23] J.Z. Guan, T. Maeda, M. Sugano, J. Oyama, Y. Higuchi, T. Suzuki, N. Makino, J. Gerontol. A Biol. Sci. Med. Sci. 63 (2008) 467-473.

[24] T. Maeda, J.Z. Guan, J. Oyama, Y. Higuchi, N. Makino, J. Gerontol. A Biol. Sci. Med. Sci. 64 (2009) 949955.

[25] G. Hudson, D. Faini, A. Stutt, M. Eccles, L. Robinson, D.J. Burn, P.F. Chinnery, Neurobiol Aging. 32(11) (2011) 2107.e3-2107.5.

[26] J. Eerola, L. Kananen, K. Manninen, O. Hellström, P.J. Tienari, I. Hovatta, J. Gerontol. A Biol. Sci. Med. Sci. 65(11) (2010) 1181-1184.

[27] C. Zhang, X. Chen, L. Li, Y. Zhou, C. Wang, S. Hou, PLoS One (2015) e0133174.

[28] E. Hiyama, K. Hiyama, T. Yokoyama, Y. Matsuura, M.A. Piatyszek, J.W. Shay, Nat. Med. 1 (1995) 249255.

[29] E. Hiyama, T. Yokoyama, N. Tatsumoto, K. Hiyama, Y. Imamura, Y. Murakami, T. Kodama, M.A. Piatyszek, J.W. Shay, Y. Matsuura, Cancer Res. 55 (1995) 3258-3262.

[30] E. Hiyama, T. Kodama, K. Shinbara, T. Iwao, M. Itoh, K. Hiyama, J.W. Shay, Y. Matsuura, T. Yokoyama, Cancer Res 57 (1997) 326-331. 
[31] E. Hiyama, K. Hiyama, Oncogene 21 (2002) 643-649.

[32] J.W. Shay, Cancer J. Sci. Am. 4 (1998) 26-34.

[33] N.W. Kim, M.A. Piatyszek, K.R. Prowse, C.B. Harley, M.D. West, P.L. Ho, G.M. Coviello, Wright WE, Weinrich SL, Shay JW. Science, 266 (1994) 2011-2015.

[34] C. Chadeneau, K. Hay, H.W. Hirte, S. Gallinger, S. Bacchetti, Cancer Res. 55 (1995) 2533-2536.

[35] H. Tahara, T. Nakanishi, M. Kitamoto, R. Nakashio, J.W. Shay, E. Tahara, G. Kajiyama, T. Ide, Cancer Res. 55 (1995) 2734-2736.

[36] H. Kojima, O. Yokosuka, F. Imazeki, H. Saisho, M. Omata, Gastroenterology 112 (1997) 493-500.

[37] W.E. Wright, J.W. Shay, M.A. Piatyszek, Nucleic Acid Res. 23 (1995) 3794-3795.

[38] M. Engelhardt, P. Drullinsky, J. Guillem, M.A. Moore, Clin. Cancer Res. 3(11) (1997) 1931-41.

[39] A.A. Avillion, M.A. Piatyszek, J. Gupta, J.W. Shay, S. Bacchetti, C.W. Greider, Cancer Res. 56 (1996) 645-650.

[40] H. Kuniyasu, T. Domen, T. Hamamoto, H. Yokozaki, W. Yasui, H. Tanara, Jpn. J. Cancer Res. 88 (1997) 103-107.

[41] T.A. Augustine, M. Baig, A. Sood, T. Budagov, G. Atzmon, J.M. Mariadason, S. Aparo, R. Maitra, S. Goel, Br. J. Cancer 112(2) (2015) 313-318.

[42] N. Tatsumoto, E. Hiyama, Y. Murakami, Y. Imamura, J.W. Shay, Y. Matsuura, T. Yokoyama, Clin. Cancer Res. 6(7) (2000) 2696-701.

(C)2015 by the authors; licensee IAPC, Zagreb, Croatia. This article is an open-access article distributed under the terms and conditions of the Creative Commons Attribution license(http://creativecommons.org/licenses/by/3.0/) (cc)) EY 